Radial and Nonradial Pulsations as Probes of Stellar Physics

ASP Conference Series, Vol. 259, 2002

C. Aerts, T.R. Bedding, 8 J. Christensen-Dalsgaard, eds.

\title{
Radial Velocity Studies of roAp stars: Rotational Modulation in HR $1217^{1}$
}

\author{
A. P. Hatzes \\ Thüringer Landessternwarte Tautenburg, Germany \\ D. Mkrtichian \\ Astronomical Observatory, Odessa, Ukraine
}

A. Kanaan

Departamento de Física, Universidade Federal de Santa Caterina, Brazil

\begin{abstract}
We present the preliminary results of our analysis of the precise radial velocity (RV) measurements of the roAp star HR 1217. We have obtained over 2000 spectra of this star ( 54 hours of coverage) covering a complete rotation period using the 2-d coude echelle spectrograph of the McDonald Observatory's 2.7m telescope. Our RV data show rotational modulation in both the amplitude and phase of the pulsations. The broad-band (covering $110 \AA$ ) RV semi-amplitudes of the $\mathrm{f}_{2}$ mode $(=2.65$ $\mathrm{mHz}$ ) varied in the range of $25 \mathrm{~m} \mathrm{~s}^{-1}$ to $200 \mathrm{~m} \mathrm{~s}^{-1}$.
\end{abstract}

\section{Introduction}

HR 1217 is a rapidly oscillating Ap star with 6 photometric periods. Most studies of roAp oscillations have used photometry, but now radial velocity measurements are providing additional information about the vertical structure of these pulsations. Here we present precise stellar radial velocity (RV) measurements for this star made with an iodine absorption cell.

\section{Observations}

Observations spanning a complete rotation period were made for HR 1217 during 16 - 22 Dec 1997 and 30 Jan - 2 Feb 1998 using the 2-d coude echelle spectrograph of the McDonald Observatory $2.7 \mathrm{~m}$ telescope. This instrument provided a wavelength coverage of 4800-6500 $\AA$ at a spectral resolution of $0.11 \AA$. Over 2000 spectra (50 sec exposures) were obtained representing a total time coverage of 54 hours. Precise stellar RVs were made using an iodine absorption cell placed in the optical path of the spectrograph. This self-calibrating technique provided a measurement accuracy of about $10 \mathrm{~ms}^{-1}$.

\footnotetext{
${ }^{1}$ This work is based on data taken at McDonald Observatory
} 


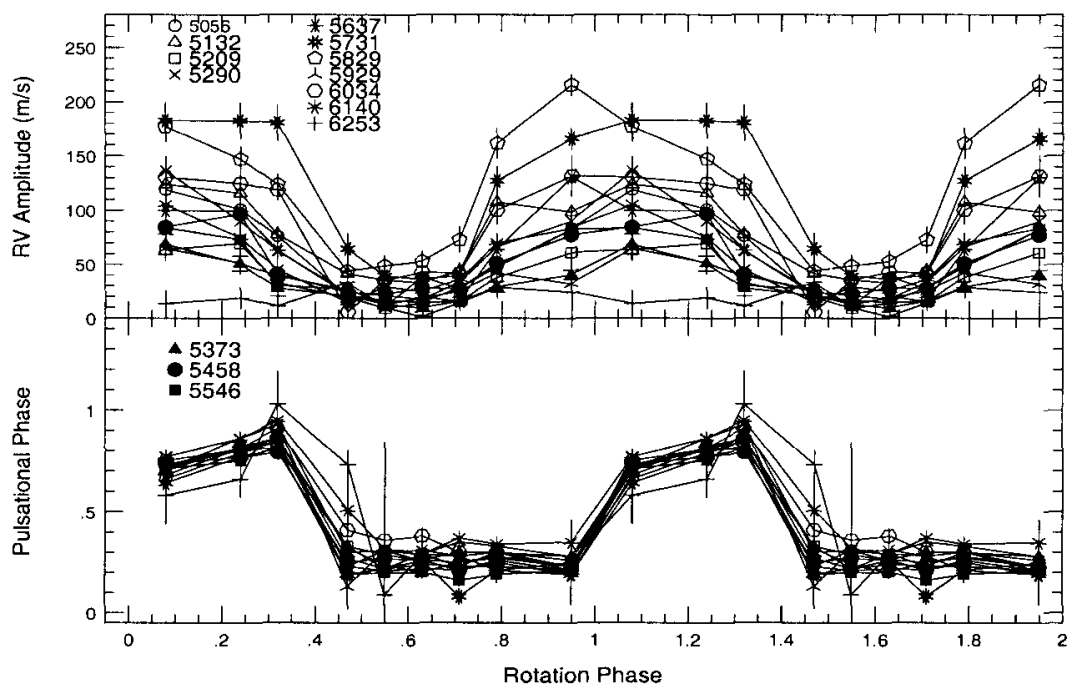

Figure 1. (top) The RV semi-amplitude of the $f_{2}$ mode $(2.65 \mathrm{mHz})$ versus rotation phase in HR 1217 in 14 spectral orders. (bottom) The pulsational phase as a function of rotation phase. The number by each symbol represents the central wavelength in $\AA$ for the spectral order.

\section{Results}

RVs were calculated using each separate spectral order (110 $\AA$ coverage in each order) of the echelle. In most spectral regions we were able to detect all 6 pulsational frequencies. Fig. 1 shows both the amplitude and phase for the $f_{2}$ mode $(=2.65 \mathrm{mHz})$ versus rotation phase. Phase 0 corresponds to a maximum in both the photometric and magnetic variations. The $f_{2}$ mode has its highest amplitude $\left(200 \mathrm{~m} \mathrm{~s}^{-1}\right)$ at $5829 \AA$ and its lowest amplitude $\left(25 \mathrm{~m} \mathrm{~s}^{-1}\right)$ at $6253 \AA$.

\section{Conclusions and future work}

We have detected rotational modulation in both the phase and amplitude of the pulsations in HR 1217 using broad-band spectral regions. The pulsational amplitude at a given rotation phase varies considerably with spectral region and is due to the fact that lines of different species can have significantly different amplitudes. We are currently performing a detailed line-by-line RV analysis of all spectral features in HR 1217. This includes not only RV determinations, but an accurate line identification and atmospheric modeling as well. This work should reveal information about the vertical structure of the pulsations in HR 1217. 